\title{
MODELO DE DESARROLLO LOCAL PARA UNA ZONA DE BOGOTÁ-COLOMBIA, RELACIONANDO LA ESTRUCTURA ECOLÓGICA PRINCIPAL Y EL PROCESO DE EXPANSIÓN URBANA
}

\section{MODEL OF LOCAL DEVELOPMENT FOR AN AREA OF BOGOTÁ-COLOMBIA, LINKING THE PROCESS OF URBAN EXPANSION AND ECOLOGICAL MAIN STRUCTURE}

\begin{abstract}
Adriana Posada Arrubla ${ }^{1}$, Jenny Vanessa Páez Chávez ${ }^{2}$
${ }^{1}$ Economista Agrícola, Esp. Gestión Social y Ambiental, M.Sc. Planeación Urbana y Regional, Docente-Investigadora Facultad Ingenierías. Universidad de Ciencias Aplicadas y Ambientales U.D.C.A, calle 222 \# 55-37, Bogotá, D.C., Colombia, e-mail: aposada@udca.edu.co; ${ }^{2}$ Joven investigadora U.D.C.A, Ingeniera Geógrafa y Ambiental U.D.C.A, calle 222 No.55-37, Bogotá, D.C., Colombia, e-mail: vanepaez1017@gmail.com
\end{abstract}

Rev. U.D.C.A Act. \& Div. Cient. 19(2): 445-455, Julio-Diciembre, 2016

\section{RESUMEN}

Dada la importancia de asumir el reto del crecimiento acelerado de las ciudades, surge la necesidad de planear estrategias de mejoramiento territorial, que contengan enfoques cruzados, capaces de interpretar la complejidad generada entre los procesos de expansión y los ecosistemas naturales. Para ello, el objetivo de este estudio fue establecer las relaciones entre la estructura ecológica principal, como componente fundamental de la ciudad de Bogotá, Colombia y la expansión urbana, como proceso de crecimiento acelerado, característico de las últimas dos décadas. Al utilizar un método con énfasis en relaciones complejas, se logró diseñar un modelo de desarrollo local, con criterios de índole multiespacial, multitemporal y multiobjetivo, que fue ajustado con la participación de las comunidades de dos barrios y con el uso de sistemas de información geográfica.

Palabras clave: Complejidad territorial, relaciones socioambientales, modelación territorial.

\section{SUMMARY}

Given the importance of the challenge of the rapid growth of cities, there is a need to generate territorial improvement strategies that contain cross approaches, able to interpret the complexity generated between processes of expansion and natural ecosystems. Therefore the aim of this study was to establish the relationships between the main ecological structure, as a fundamental component of urban expansion, and the city of Bogotá - Colombia, as a process of accelerated growth characteristic of the last two decades. When employing a method with emphasis on complex relationships, the design of a model of local development was achieved, with criteria of multi-spatial, multi-temporal and multi-objective nature, adjusted with the participation of the communities in two districts and the use of geographic information systems.

Key words: Territorial complexity, social and environmental relations, territorial modeling.

\section{INTRODUCCIÓN}

Las problemáticas ambientales del proceso de expansión urbana son ya un factor común mundial y ello incide en un cambio de análisis y de perspectivas, para mejorar las políticas públicas y los modelos de desarrollo, tal como ha sucedido en Europa, donde no se mantiene ya, como única pretensión, conservar intactos los paisajes naturales, sino orientar su evolución, mediante instrumentos de gestión y de ordenación (Lahoz, 2010). Este asunto es tratado por el PNUMA (2012), con su posición mundial, para definir nuevos escenarios, en función de la transformación de la sostenibilidad. En Latinoamérica, la situación de los impactos de la expansión urbana sobre los componentes ambientales estructurantes, también son abordados como una premisa del desarrollo territorial; por ejemplo, en México, los efectos ambientales de la expansión urbana, rebasan los límites de la ciudad, ejerciendo presión sobre zonas circunvecinas y, por ello, la ciudad no puede ser vista como un sistema autosuficiente (Pisanty et al. 2009). Por su parte, Colombia y 
en especial su ciudad central Bogotá, no han sido ajenos al estudio y adaptación de políticas y estrategias frente a esta problemática, pero dada su complejidad, un estudio más al respecto, puede arrojar nuevas luces para su tratamiento.

El crecimiento urbano en las principales ciudades del mundo ha sido acelerado y se ha aumentado la densidad de población, debido, principalmente, a migraciones de habitantes rurales, generando expansión de las urbes en un tiempo relativamente corto, dejando el $80 \%$ de la población en ciudades (ONU-Habitat, 2012). En capitales como Bogotá, su modelo de desarrollo previo y de planificación, generó un crecimiento informal y periférico, que consolidó zonas de riesgo para la población y para la estructura natural de la urbe, al no considerar la capacidad del ambiente para soportar la concentración de población, de urbanizaciones y de contaminación. Esta tendencia de sobrepasar los límites geográficos ha ocasionado impactos negativos en diferentes zonas, por la excesiva demanda de recursos naturales, la generación de residuos y las emisiones contaminantes al suelo, agua y aire. La problemática de dicha situación es que el impacto podría ser el correspondiente a un espacio de mayor área, aunque la zona tenga una extensión menor (Lahoz, 2010).

La estructura ecológica principal (EEP), entendida como la "red de espacios y corredores que sostienen y conducen la biodiversidad y los procesos ecológicos esenciales, a través del territorio del Distrito Capital" (AMB, 2013), ha sido de los componentes más afectados por causa del crecimiento urbano, ya que por ser el instrumento de planificación de mayor jerarquía para la conservación y el desarrollo sostenible de la ciudad (Andrade et al. 2008) tiene incidencia en la forma de ocupación del territorio y en la población que se ve afectada, por la forma inadecuada como se integra al proceso de expansión urbana.

Teniendo en cuenta que lo que se busca es la relación armónica población-territorio por medio de diversas estrategias e instrumentos (Posada \& Berrocal, 2015), es importante comprender que la expansión urbana es una realidad que se debe asumir e integrar al concepto natural de la ciudad, porque de lo contrario, difícilmente, se logrará el desarrollo sostenible del territorio. Una de las localidades donde se presentó un incremento apresurado, tanto poblacional como territorialmente, afectando de manera directa la EEP, fue la localidad de Kennedy, considerada como la más poblada de todo el distrito, con 1.500.000 habitantes aproximadamente, ubicada en el occidente de Bogotá (ALK, 2013). En esta localidad, las zonas más afectadas por contar con mayor presencia de elementos naturales de la EEP, fueron las unidades de planeación zonal (UPZ) Calandaima y Tintal Norte. Este territorio representa una zona en desarrollo que está creciendo urbanísticamente y el posicionamiento sobre la EEP, a pesar de que está reglamentado en varios casos, es progre- sivo y está generando consecuencias e impactos negativos, ya que la afectación, la transformación y el agotamiento de los elementos característicos de dicha estructura, impide la prestación de servicios ambientales fundamentales para la población, no solo local sino regional.

Por lo anterior, se vio la importancia de establecer las relaciones existentes entre la EEP y el proceso de expansión urbana (PEU), para determinar un modelo de desarrollo local sostenible (MDLS), que permitiera, por un lado, visualizar la problemática de forma integral, a partir de las relaciones entre los aspectos físicos, espaciales, ambientales, sociales y normativos y, por otro, que posibilitara la creación de estrategias para solucionar los conflictos, incluyendo y fortaleciendo la participación comunitaria, con el fin de mitigar problemáticas actuales y prevenir las dificultades futuras. Como afirman Martínez \& Milla (2012), las estrategias del medio no surgen de la nada, por ello, para definir el modelo fue fundamental prever la tendencia de la intervención, ya que a partir de ésta, es posible determinar las condiciones a las que la situación estará sometida.

Ya que el enfoque del estudio se centró en la generación de un MDLS es importante entender que dicho modelo no necesariamente constituyó una explicación de la realidad física, ya que, según Concari (2011), representar no es describir, ni tampoco es explicar; las representaciones pueden ser vistas como medios para comprender y conocer, por lo que el modelo puede ser concebido como una representación posible de una cosa o evento; que para el presente estudio, se visualizó como una solución a corto, mediano y largo plazo, de las condiciones actuales del territorio estudiado. La especificad de desarrollo local y la parte de la sostenibilidad, se enfocó en relación con el bienestar, el crecimiento y el progreso (Sanchis, 2010). La búsqueda del mejoramiento de calidad de vida de la población, por medio del crecimiento económico, se enfocó en la potencialidad territorial (Gaviria, 2009). Todo ello, en el marco de una visión de largo plazo, para establecer una serie de políticas de gestión, que permitan alcanzar la gobernanza democrática del territorio (Bravo \& Marín, 2014).

En la zona de estudio, el incremento poblacional acelerado, a finales del siglo pasado, generó cambios ambientales y urbanos, especialmente, en las formas de construcción de vivienda, pasando de ser un territorio con ciertas características rurales, a ser un paisaje netamente urbano. La problemática, se evidenció hasta que la oferta de vivienda dejó de ser suficiente para suplir la demanda y aparecieron las construcciones informales, en sitios no aptos para urbanizar, con riesgos para las edificaciones y la población residente en ellas, multiplicando las problemáticas naturales y a la población, en general. Dada la importancia de la sostenibilidad en 
zonas locales, el objetivo del presente escrito es difundir la construcción de un modelo de desarrollo local, a partir de la relación entre la EEP y el PEU de las UPZ Calandaima y Tintal Norte, de la capital colombiana.

\section{MATERIALES Y MÉTODOS}

La investigación, se desarrolló en las UPZ Calandaima y Tintal Norte, ubicadas en la localidad de Kennedy, al sur occidente de Bogotá (Mapa 1). La caracterización de la EEP y del PEU, se efectuó mediante una verificación teórica y normativa; además, se utilizaron aerofotografías, para la elaboración de la cartografía base. Respecto a la EPP, se listaron y se explicaron todos sus elementos y para el PEU, se realizó un estudio multi-temporal, empleando fotografías aéreas de 1998, del Instituto Geográfico Agustín Codazzi e imágenes de Google Earth, de 2006 y de 2014. La cartografía correspondiente de 1998 fue elaborada mediante diferentes fotografías aéreas, que conforman la totalidad de la zona de estudio, realizando un mosaico, para obtener una sola fotografía, que contuviera las dos UPZ.

Dado que el modelo parte de la identificación de las relaciones entre la EEP y el PEU, este procedimiento se llevó a cabo

Mapa 1. Proceso histórico de expansión urbana de las UPZ Calandaima y Tintal Norte, en Bogotá, Colombia.

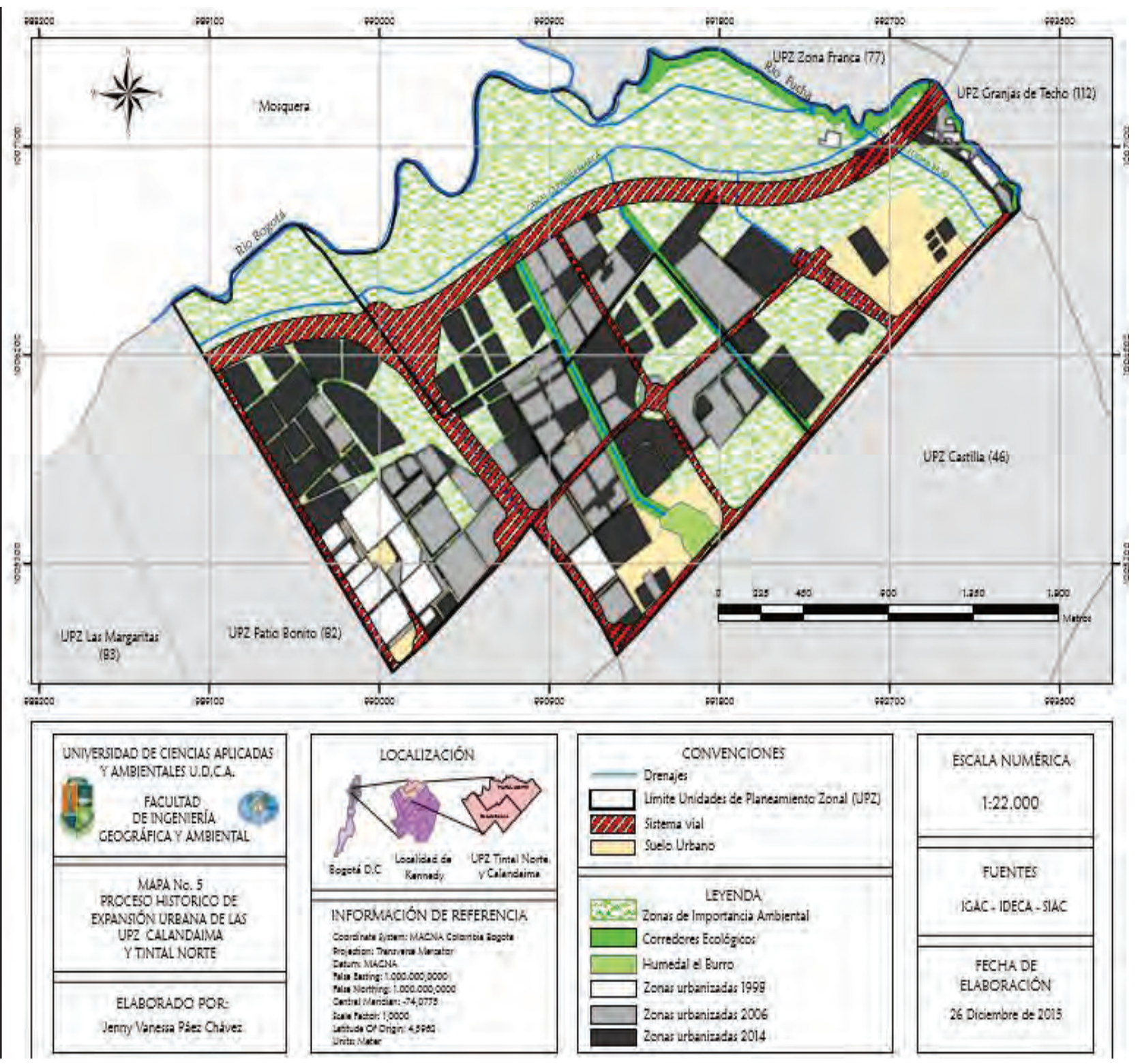


en cuatro etapas: Para las relaciones espaciales, se realizó una demostración, a través de un sistema de información geográfica (SIG), evidenciando la relación física entre la EEP y el PEU en las UPZs. La etapa correspondiente a las relaciones socioambientales tuvo en cuenta las condiciones de vida de la población residente en la zona de estudio y su interacción con los elementos ambientales de la EEP; además, se utilizó el análisis de coyuntura, teniendo en cuenta la premisa de que éste permite conocer la realidad histórico-social, para orientar el cambio en función de las necesidades e intereses (Gallardo, 1990). Para la relación población-entorno y su perspectiva del territorio, se realizó una entrevista semiestructurada; esta herramienta | se ajusta adecuadamente a dicho propósito, ya que se considera indicada para objetivos descriptivos, donde se requiere saber algún aspecto específico de la población (Ochoa, 2013). La fórmula para determinar la muestra de la población a entrevistar fue la siguiente:

$$
n=\frac{N * Z^{2} * p *(1-p)}{(N-1) * e^{2}+Z^{2} * p *(1-p)}
$$

Dónde: $n=$ Tamaño de la muestra; $N=$ Cantidad de población (personas entrevistadas en 12 barrios); $Z=$ Nivel de confianza (95\%, que equivale a $Z=1,96) ; p=$ Proporción esperada $(50 \%=0,5)$ y $e=$ Margen de error admitido $(5 \%$ $=0,05)$ :

$$
\begin{gathered}
n=\frac{23 * 1,96^{2} * 0,5 *(1-0,5)}{(23-1) * 0,05^{2}+1,96^{2} * 0,5 *(1-0,5)} \\
n=\frac{22.0892}{1.0154} \\
n=21.75418554 \sim 22
\end{gathered}
$$

Este resultado hace referencia a la cantidad de habitantes que se requieren entrevistar y se decidió duplicarlo, para entrevistar a dos habitantes por barrio, en cada una de las dos localidades, para un total de 44 habitantes, a quienes se aplicaron 15 preguntas, sobre la situación pasada y presente de las condiciones del territorio, las principales problemáticas ambientales y sociales, y la forma como la población relaciona dichas situaciones.

La etapa participativa consistió en la realización de un taller con la comunidad, para generar el modelo de desarrollo local. Esta actividad estuvo compuesta de los siguientes pasos: (I) identificación de los usos del suelo en la zona de estudio; (II) clasificación de las variables que intervienen en el desarrollo; (III) identificación de ejes estratégicos de desarrollo local sostenible; (IV) diseño del MDLS, explicación teórica del MDLS, explicación esquemática del MDLS y elaboración cartográfica, que explica el diseño del MDLS.

\section{RESULTADOS Y DISCUSIÓN}

Este estudio evidencia que en las UPZ Calandaima y Tintal Norte existe un deterioro constante de los elementos de la EEP; es débil e inexistente, en algunos casos, y carece de la capacidad de cumplir funciones, como conectar las zonas de importancia ambiental, generar sostenibilidad, conducción de procesos ecológicos, conservar los recursos naturales y aumentar las condiciones de habitabilidad de la población.

\section{Sobre la identificación de relaciones}

Relaciones espaciales. Las relaciones espaciales contribuyen con el entendimiento de la interacción entre los componentes del territorio y la manera en que generan vínculos importantes para el desarrollo y la continuidad del mismo. Estas relaciones permiten representar la realidad y observar la organización del territorio, teniendo en cuenta los factores que lo constituyen. El procedimiento para identificar las relaciones de tipo espacial, se llevó a cabo por medio de la realización del mapa 2 y el cuadro 1 (parte A), donde se visualizan las alteraciones y las modificaciones espaciales en el territorio, que ocasionan el inicio de nuevos procesos, para acondicionar a la población a los sucesos futuros. Las acciones o las situaciones sucesivas en el tiempo, crearon unas relaciones entre la EEP y el PEU de las UPZs, que se pueden clasificar en 10 tipos.

Relaciones socioambientales. La población residente y flotante de las UPZ Calandaima y Tintal Norte tiene una perspectiva del territorio positiva, en general, pero es consciente que existen muchas problemáticas que no permiten que se genere un desarrollo local óptimo. El total de la población entrevistada ha observado cambios físicos significativos, independientemente del tiempo que lleva viviendo allí y han evidenciado que el PEU se ha desarrollado en, relativamente, poco tiempo y debido a la implementación de las nuevas edificaciones, se asume que la tendencia es que este crecimiento continúe hacia las zonas que aún no están construidas. La población reconoce que es la principal responsable por la falta de consciencia, tolerancia y, en algunos casos, por el desconocimiento de las consecuencias, pero aun así no busca la manera que estas relaciones cambien o mejoren. Esta responsabilidad también se le atribuye a las entidades sociales y ambientales, encargadas de gestionar las mejoras y el acompañamiento a la población.

Teniendo en cuenta la perspectiva de la población frente al territorio y el análisis de la forma de concebir el lugar en el que habitan, se identificaron 10 tipos de relaciones sociambientales, que se describen en el cuadro 1 (parte B). En la figura 1, se muestra de forma sistémica, las relaciones identificadas y los elementos en conexión con el resto del territorio, demostrando que más allá de la extensión que estos posean o de su capacidad de generar diversos procesos, la 
Mapa 2. Relaciones espaciales de las UPZ Calandaima y Tintal Norte, en Bogotá, Colombia.

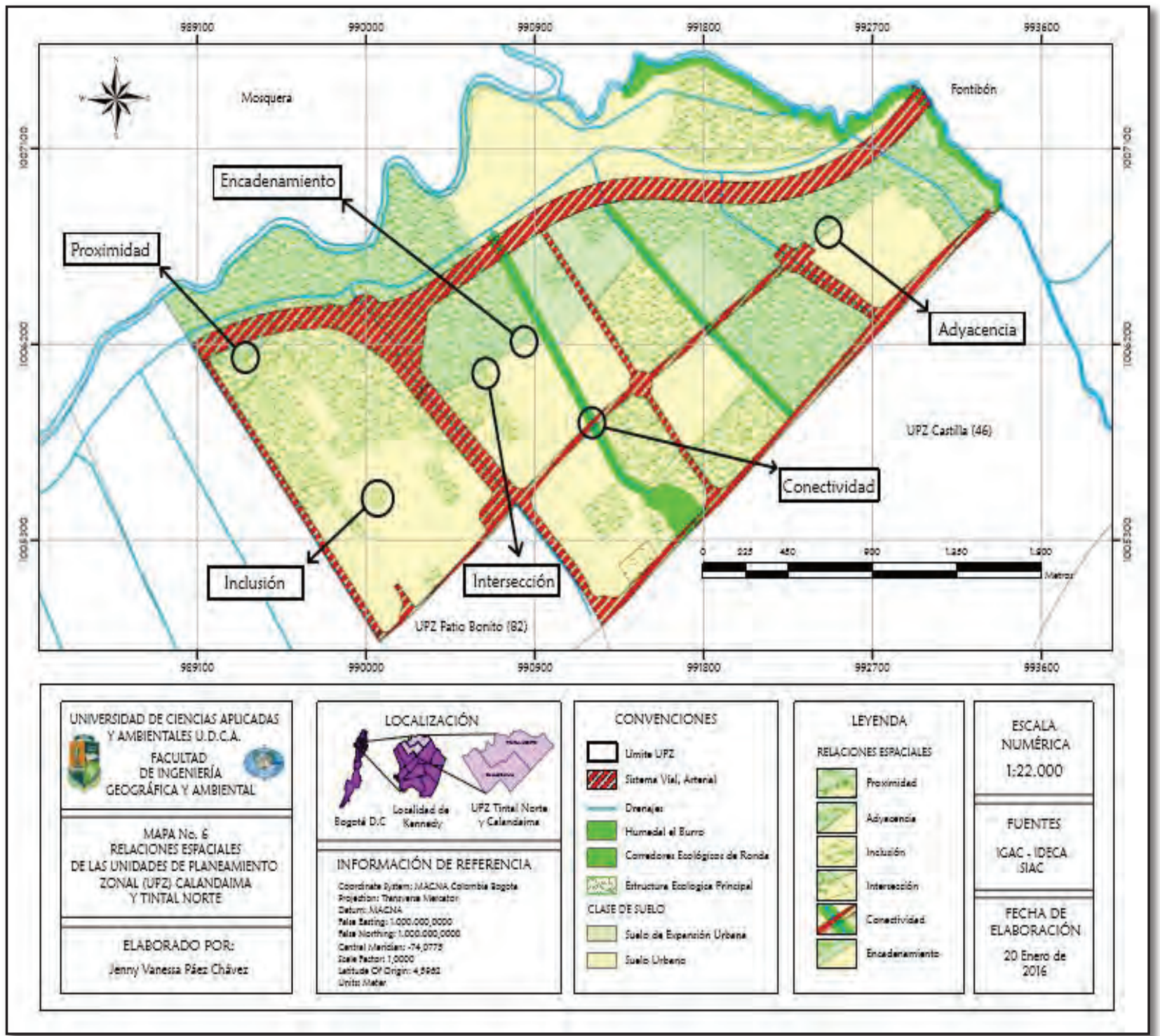


Cuadro 1. Clasificación de las relaciones: A (Espaciales) y B (Socioambientales).

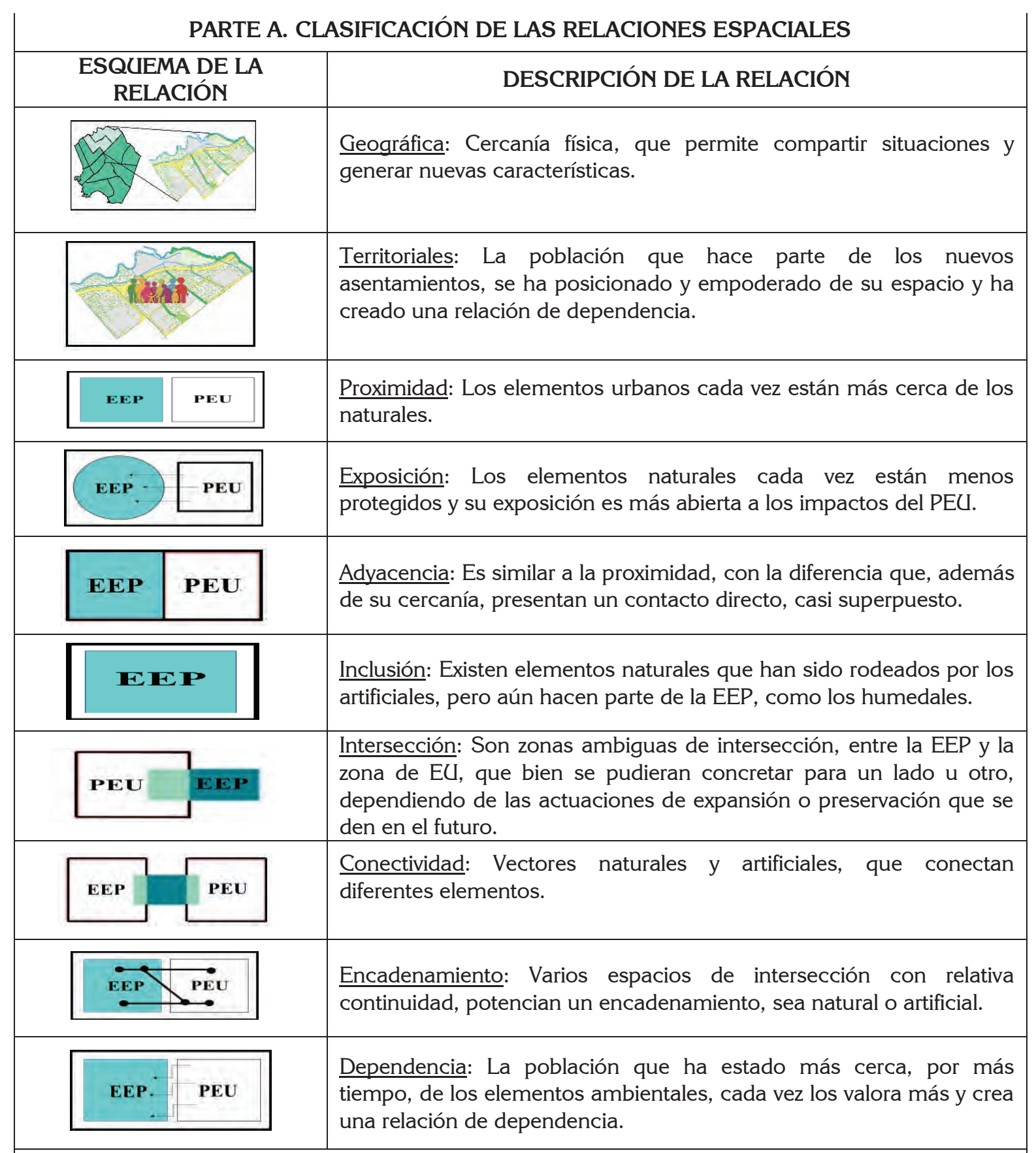




\begin{tabular}{|c|c|}
\hline \multicolumn{2}{|c|}{ PARTE B. CLASIFICACIÓN DE LAS RELACIONES SOCIOAMBIENTALES } \\
\hline TIPO DE RELACIÓN & DESCRIPCIÓN \\
\hline ESTRECHA & $\begin{array}{l}\text { Debido a la cercanía de la EPP con la expansión urbana, se crea una relación } \\
\text { estrecha, porque los elementos no se desarrollan en espacios extensos, sino en } \\
\text { pequeñas proporciones, donde la intervención o la modificación de cualquier } \\
\text { elemento, tiene repercusión en todo lo que se encuentra alrededor. }\end{array}$ \\
\hline DESPROPORCIÓN & $\begin{array}{l}\text { Existe una desproporción entre el área que corresponde a la EEP y el posicion- } \\
\text { amiento de la expansión urbana sobre la EEP, que ocasiona que esta pierda } \\
\text { extensión y que tienda a desaparecer por completo. }\end{array}$ \\
\hline DEPENDENCIA & $\begin{array}{l}\text { Esta relación, se evidencia por la necesidad de la expansión urbana por construir } \\
\text { en espacios que cuenten con elementos de la EEP y por la necesidad de la po- } \\
\text { blación, por contar con los servicios ambientales que ofrece la EEP. }\end{array}$ \\
\hline DESIGUALDAD & $\begin{array}{l}\text { La prioridad que se la ha dado a la zona de expansión urbana ocasionó que los } \\
\text { intereses por preservar los elementos de la EEP disminuyeran, ocasionando una } \\
\text { inclinación por mejorar las zonas construidas y desmejorar la EEP. }\end{array}$ \\
\hline ANTAGÓNICA & $\begin{array}{l}\text { Manejar los elementos del territorio por separado ocasiona que se generen rela- } \\
\text { ciones con intereses opuestos, donde prima el bienestar para lo particular y no } \\
\text { para lo general. }\end{array}$ \\
\hline INTERESES & $\begin{array}{l}\text { La consolidación de la población sobre las zonas de expansión urbana género } \\
\text { que las personas se interesaran por estar cerca de elementos de la EEP, debido a } \\
\text { los servicios ambientales que ofrecen y el bienestar que mejora las condiciones } \\
\text { de vida. }\end{array}$ \\
\hline REFLEXIVA & $\begin{array}{l}\text { Por la presencia de la población en las zonas de expansión urbana, se genera } \\
\text { una relación reflexiva frente a la percepción de las personas sobre la EEP, ya que } \\
\text { estar tan cerca de sus elementos origina una introspección sobre su importan- } \\
\text { cia y la necesidad de preservarlos, en óptimas condiciones. }\end{array}$ \\
\hline COMPROMISO & $\begin{array}{l}\text { La generación de las relaciones reflexivas permitió el incremento de compro- } \\
\text { miso por cuidar y preservar los elementos de la EEP, debido al bienestar que } \\
\text { brinda para la población local y por el aumento de la calidad del ambiente, que } \\
\text { mejora las condiciones del entorno en general. }\end{array}$ \\
\hline COOPERATIVAS & $\begin{array}{l}\text { El hecho de que se forje un compromiso por respetar y preservar los elementos } \\
\text { de la EEP por parte de la población asentada en las zonas de expansión urbana, } \\
\text { genera una unión entre la población por ejecutar acciones, que permitan la } \\
\text { conservación y la protección de dichos elementos. }\end{array}$ \\
\hline COLECTIVAS & $\begin{array}{l}\text { El incremento del interés por proteger las zonas de importancia ambiental, a } \\
\text { pesar de la presencia de asentamientos urbanos, fomenta conexiones, no solo } \\
\text { con la población sino con actores externos, que puedan contribuir con el mejo- } \\
\text { ramiento de las condiciones locales. }\end{array}$ \\
\hline
\end{tabular}




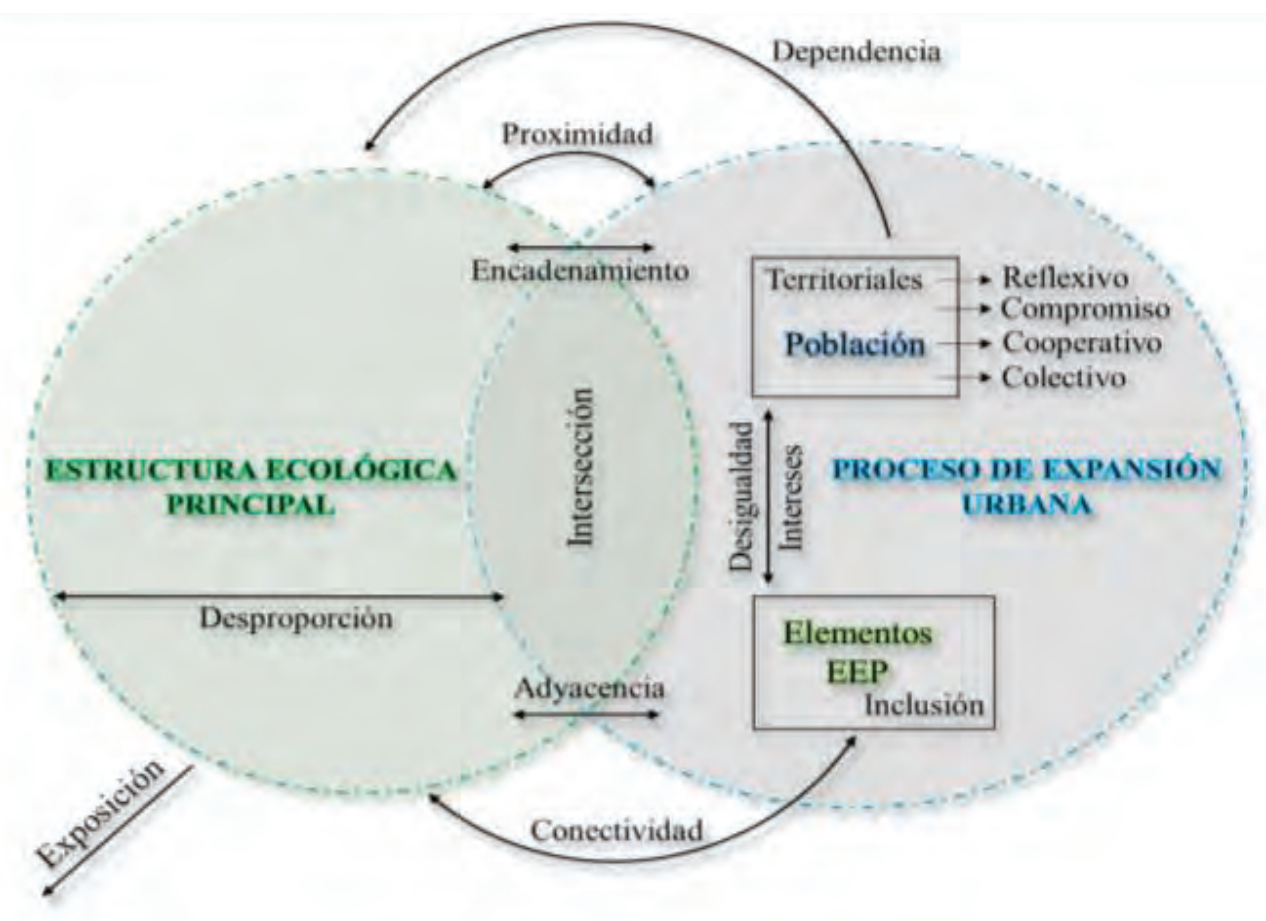

Figura 1. Resultado de relaciones entre la estructura ecológica principal y el proceso de expansión urbana, en Bogotá, Colombia.

transformación o cambio de alguno de ellos altera representativamente la funcionalidad de todo el sistema que, en este caso, se refiere a las UPZ Calandaima y Tintal Norte.

\section{Construcción del modelo de desarrollo local}

A partir de las relaciones identificadas en conjunto con la comunidad, se agruparon los resultados, con el fin de considerar la posibilidad de fortalecimiento de cada relación y el lapso de tiempo requerido para llevarlo a cabo. Asimismo, se consideró importante incluir las oportunidades y las amenazas, consideradas por la población, para que las estrategias de desarrollo local sostenible se enfocaran en incrementar las oportunidades y transformar las amenazas en situaciones positivas. Posteriormente, se construyó el modelo, traslapando las diferentes escalas espaciales y temporales y, en razón al cruce entre éstas, se trazaron unos objetivos, para el logro progresivo y gradual del modelo de desarrollo local.

Definición de la escala espacio-temporal. La escala espacial, se determinó teniendo en cuenta los requerimientos planteados en la escala temporal, donde el corto plazo, incluye a la UPZ Calandaima; el mediano plazo, a la UPZ Calandaima y Tintal Norte y el largo plazo, a la UPZ Calandaima,
Tintal Norte y los barrios Osorio, Castilla, Pio XII y las Dos Avenidas (Figura 2).

Definición de objetivos. Para lograr que las UPZ Calandaima y Tintal Norte se consoliden localmente es necesario trazar objetivos de manera gradual y consecutiva, así:

En el corto plazo: Fortalecer las relaciones positivas, que permiten que los elementos que componen el territorio se desarrollen de manera adecuada, por medio de la consolidación, la creación y la integración de grupos sociales y actores que intervienen en la UPZ Calandaima.

En el mediano plazo: Modificar las condiciones negativas actuales del territorio, por medio del mejoramiento de las relaciones entre los elementos que lo componen, teniendo en cuenta los dos componentes principales de las UPZ Calandaima y Tintal Norte; la EEP y el PEU.

En el largo plazo: Armonizar las dinámicas territoriales, por medio de la integración sostenible de los elementos del territorio, conformado por las UPZ Calandaima y Tintal Norte y barrios Castilla. 


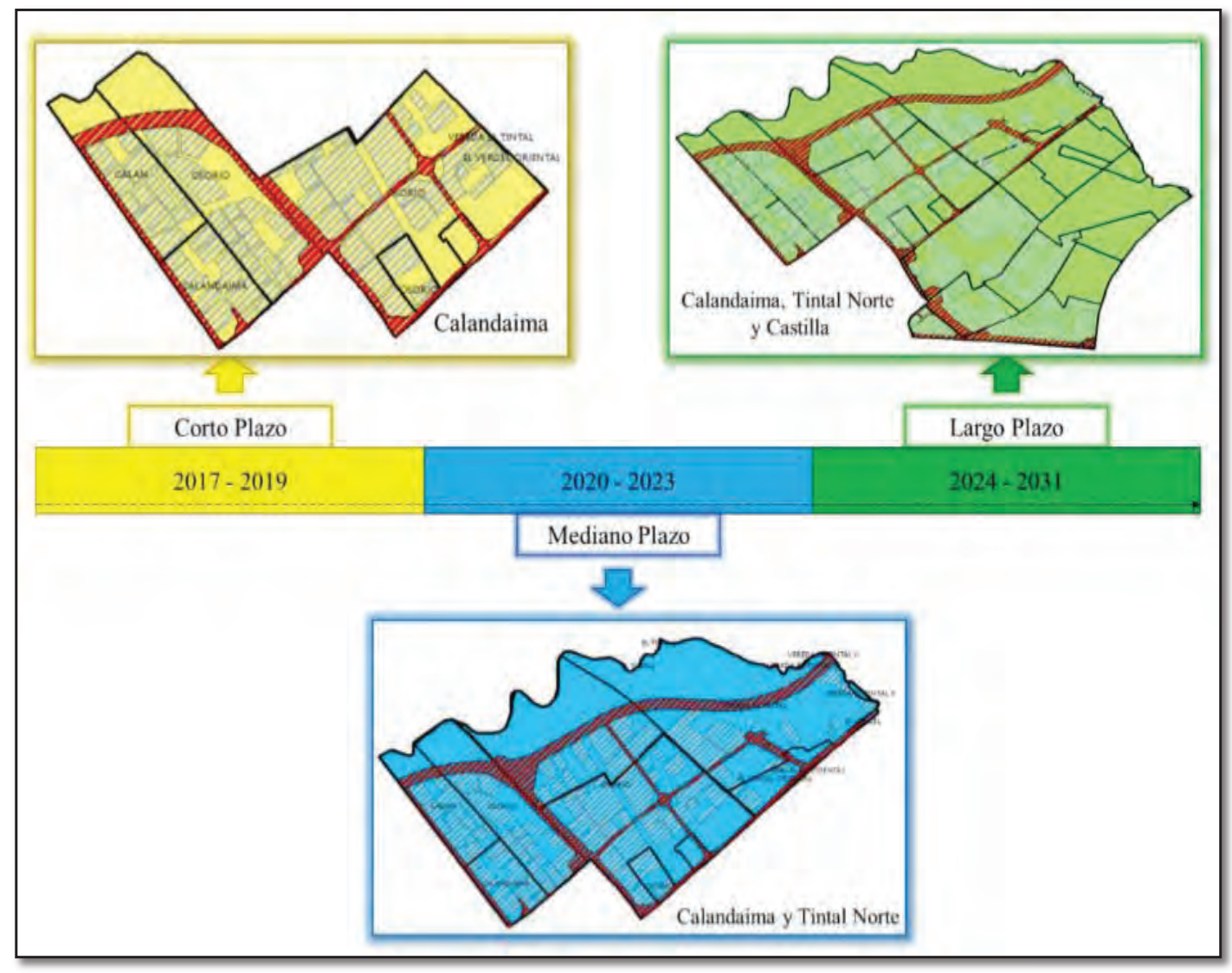

Figura 2. Representación de la escala espacio temporal del modelo de desarrollo local sostenible, en Bogotá, Colombia.

En el cuadro 2, se presenta una síntesis dinámica del modelo, donde se explican las condiciones principales que se tuvieron en cuenta para el diseño de esta alternativa de desarrollo del territorio local. Este modelo es acorde, no solo a las condiciones de las UPZ Calandaima y Tintal Norte sino que relaciona otros espacios que tienen que ver, principalmente, con la afectación a las fuentes hídricas y los ecosistemas que conectan, por lo que se incluyen elementos de la EEP de la UPZ Castilla, que se encuentran impactados en mayor proporción por la población de esta UPZ, con influencia sobre el canal que lleva sus aguas a toda la red hídrica de las UPZ Calandaima y Tintal Norte.

Por medio de este ejercicio, se logra comprender que el diseño prospectivo es un reto importante en las soluciones de los problemas territoriales, debido a la exigencia para concretar la capacidad de contextualizar, no solo el problema sino la alternativa de solución, para el presente y el futuro; dado que los problemas territoriales más complejos se presentan en las interfaces de situaciones, de espacios territoriales y de tendencias, justo aquí es donde se puede plantear y actuar sobre modelos de desarrollo.
Los problemas territoriales son tantos, tan grandes, profundos y difusos, que requieren soluciones muy concretas, puntuales e innovadoras, como lo es el diseño de modelos de desarrollo local. Según Posada Arrubla et al. (2016), un modelo puede ser una representación esquemática de los elementos de un sistema y de las relaciones que existen entre ellos, por lo cual, la modelación territorial exige detallar los procesos de integración, de relaciones e interacciones externas e internas del sistema territorial.

En consonancia con lo anterior, para la FAO (s/f), en América Latina y el Caribe, el problema de las desigualdades socioespaciales, se identifica como un aspecto central del ordenamiento territorial y en todos los países destaca la necesidad de lograr mayor cohesión territorial, perspectiva, bajo la cual, identifica que existen dos corrientes en los enfoques territoriales latinoamericanos: el ambientalista y el de desarrollo local.

En el modelo aquí expuesto, se combina el enfoque ambientalista y el enfoque de desarrollo local, agrupándolo en el desarrollo local sostenible. Lo que se busca, entonces, es 
Cuadro 2. Síntesis del modelo de desarrollo local sostenible.

\begin{tabular}{|c|c|c|c|}
\hline Temporalidad & $\begin{array}{c}\text { Corto Plazo } \\
(2017-2019)\end{array}$ & $\begin{array}{r}\text { Mediano Plazo } \\
(2020 \text { - 2023) }\end{array}$ & $\begin{array}{c}\text { Largo Plazo } \\
(2024-2031)\end{array}$ \\
\hline $\begin{array}{c}\text { UPZ } \\
\text { Calandaima }\end{array}$ & Fertalecimiento & $\begin{array}{c}\text { Desarrollo del } \\
\text { fortalecimiento }\end{array}$ & $\begin{array}{l}\text { Prolongación del } \\
\text { fortalecimiento }\end{array}$ \\
\hline $\begin{array}{c}\text { UPZ } \\
\text { Calandaima y } \\
\text { Tintal Norte } \\
\end{array}$ & $\begin{array}{l}\text { Alternativas de } \\
\text { mejoramiento }\end{array}$ & Mejoramiento & $\begin{array}{l}\text { ad del } \\
\text { iento }\end{array}$ \\
\hline $\begin{array}{c}\text { UPZ } \\
\text { Calandaima, } \\
\text { Tintal Norte y } \\
\text { Castilla }\end{array}$ & $\begin{array}{l}\text { Transición entre } \\
\text { la planeación yla } \\
\text { ejecución }\end{array}$ & Ejecución & Cor \\
\hline - & $\begin{array}{lr}\text { Fortalecer } & \text { las } \\
\text { situaciones } & \\
\text { positivas que } & \text { que } \\
\text { permiten que los } \\
\text { elementos que } \\
\text { componen el } \\
\text { territorio se } \\
\text { desarrollen de } \\
\text { manera } \\
\text { adecuada, por } \\
\text { medio de la } \\
\text { consolidación, } \\
\text { creación e } \\
\text { integración de } \\
\text { grupos sociales y } \\
\text { actores que } \\
\text { intervienen en la } \\
\text { UPZ Calandaima. }\end{array}$ & $\begin{array}{l}\text { Modificar } \\
\text { condiciones } \\
\text { actualas } \\
\text { territorio, del } \\
\text { medio } \\
\text { mejoramients de } \\
\text { las relacilnes } \\
\text { entre } \\
\text { elementos que lo } \\
\text { componen, } \\
\text { teniendo } \\
\text { cuenta los dos } \\
\text { componentes } \\
\text { principales de las } \\
\text { UPZ Calandaima y } \\
\text { Tintal Norte; la } \\
\text { EEP y el PEU. }\end{array}$ & 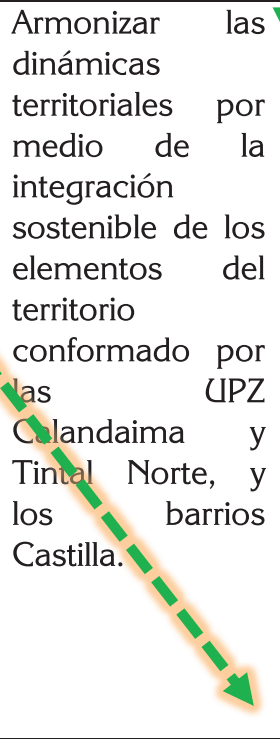 \\
\hline
\end{tabular}

armonizar las dinámicas territoriales, por medio de la formulación y la ejecución de políticas, de planes, de programas y de proyectos, con el fin de realizar una gestión del territorio; sin embargo, el presente modelo no diseña estas acciones específicas, pero sí genera una serie de estrategias, que se derivan de un proceso histórico y natural, para orientar dichas acciones.

Los fundamentos del desarrollo local sostenible del modelo contiene, por un lado, la integración y el refuerzo de las relaciones entre los diversos actores y, por otro, los elementos naturales. También incluye el cumplimiento normativo, para impulsar la importancia de actuar, conforme a las normas y aumentar el seguimiento y el control normativo, lo que permite contemplar la posibilidad de ajustar el marco normativo, para regular las relaciones entre la EEP y las actuaciones urbanísticas en las UPZ Calandaima y Tintal Norte.

Con este modelo, se hace evidente la importancia de integrar a la comunidad en todos los procesos de planeación, de formulación y de gestión, que se llevan a cabo en el territorio. Y dado que el ordenamiento territorial es un proceso que utiliza diversas estrategias e instrumentos para lograr una relación armónica población-territorio (Posada A. \& Berrocal, 2015), en el diseño de modelos, se exige contar con herramientas, que permitan establecer diferentes alternativas frente a una problemática territorial.

En conclusión, es importante generar un equilibrio entre el uso que le da la población al entorno natural y la forma como se retornan los servicios que ofrece la naturaleza, ya que un defecto que ha caracterizado a la población, en general es que sobreutiliza y explota los recursos naturales y no se preocupa por hacer procesos de recuperación al ambiente.

La relación entre la EEP y el PEU, se ha hecho más evidente con el transcurso de los años, ya que se encuentra inmersa una en la otra y hacen parte de la composición del territorio, en general. Frente a lo cual, es esencial analizar cada parte del territorio, pero resulta fundamental observar las relacio- 
nes existentes entre los elementos, puesto que las manifestaciones particulares tiene repercusiones, a nivel general y se hace necesario comprender al territorio como un sistema complejo, que no se puede manejar por separado.

La identificación de relaciones permitió comprobar las diferentes formas y situaciones, por medio, de las cuales, los elementos del territorio tienen alguna conexión o generan algún tipo de vínculo. Por medio del conocimiento de las relaciones que existen en el territorio es posible evidenciar las consecuencias de sus elementos, suscitadas por alteraciones previas. Así, para planificar el uso, el manejo y la ocupación del territorio es importante hacerlo de forma integral, teniendo en cuenta no solo dichos elementos sino las razones por las cuales se generan procesos, que conducen al desarrollo.

Se sugiere, por lo tanto, que para observar el territorio como un todo, se debe mantener rigurosidad en buscar soluciones prácticas, que se puede lograr trazando el siguiente proceso, conducente al desarrollo: Identificar sistemas territoriales en conflicto; entender la estructura de los sistemas territoriales en conflicto; abstraer detalles sobre la estructura de los sistemas territoriales en conflicto; DISEÑAR MODELOS PROSPECTIVOS DE ORDENAMIENTO PARA EL DESARROLLO TERRITORIAL; sugerir políticas de intervención para los sistemas territoriales en conflicto e intervenir los sistemas territoriales en conflicto.

Conflicto de intereses: El manuscrito fue preparado y revisado con la participación de las autores, quienes declaramos que no existe conflicto de intereses que ponga en riesgo la validez de los resultados presentados y quienes agradecen a la U.D.C.A la financiación de la investigación "Convergencias de la Gestión Ambiental Territorial en Bogotá".

\section{BIBLIOGRAFÍA}

1. ALCALDÍA LOCAL DE KENNEDY-ALK-. 2013. Conociendo mi Localidad. Disponible desde Internet en: http://www.kennedy.gov.co/index.php/milocalidad/conociendo-mi-localidad (con acceso el 15/02/2016).

2. ALCALDÍA MAYOR DE BOGOTÁ -AMB-. 2013. Plan de Ordenamiento Territorial de Bogotá. Disponible desde Internet en: http://www.sdp.gov.co/portal/page/portal/PortalSDP/POT_2020/POT/Decreto364-2013.pdf (con acceso el 15/02/2016).

3. ANDRADE, G.; MESA, C.; RAMÍREZ, A.; REMOLINA, F. 2008. Estructura ecológica principal y áreas protegidas de Bogotá, oportunidad de integración de políticas para la construcción y el ordenamiento del territorio de la ciudad-región. Bogotá, Colombia:
Foro Nacional Ambiental. Disponible desde Internet en: http://ibrary.fes.de/pdf-files/bueros/kolumbien/06455.pdf (con acceso el 10/12/2015).

4. BRAVO, O.; MARÍN, F. 2014. Modelo de desarrollo local para los municipios. Cuaderno del CENDES. 31(86):1-26.

5. CONCARI, S. 2011. Las teorías y modelos en la explicación científica: implicancias para la enseñanza de las ciencias. Ciencia y Educación 7(1):85-94.

6. GALLARDO, H. 1990. Fundamentos de Formación Política: Análisis de Coyuntura. Santiago de Chile (Chile). $85 p$.

7. GAVIRIA, Z. 2009. La expansión urbana sobre las periferias rurales del entorno inmediato a la ciudad metropolitana. Rev. Soluciones de Postgrado EIA. 3:63-74.

8. LAHOZ, E. 2010. Reflexiones medioambientales de la expansión urbana. Cuadernos Geográficos. 46:293-313.

9. MARTÍNEZ, D.; MILLA, A. 2012. La elaboración del plan estratégico y su implantación a través del cuadro de mando integral. Ediciones Díaz de Santos. España. p.33-39.

10. OCHOA, C. 2013. Tamaño de la muestra. Disponible desde Internet en: http://www.netquest.com/blog/ es/que-tamano-de-muestra-necesito/ (con acceso el 23/02/2016).

11. ORGANIZACIÓN DE LAS NACIONES UNIDAS PARA LA ALIMENTACIÓN Y LA AGRICULTURA -FAO-. $(\mathrm{s} / \mathrm{f})$. Plataforma de territorios inteligentes. Disponible desde Internet en: http://www.fao.org/in-action/ territorios-inteligentes/componentes/ordenamientoterritorial/es/ (con acceso el 17/03/2016).

12. PISANTY, I.; MAZARI, M.; EZCURRA, E. 2009. El reto de la conservación de la biodiversidad en zonas urbanas y periurbanas, en Capital natural de México, vol. II: Estado de conservación y tendencias de cambio. Conabio, México. p.719-759.

13. PNUMA. 2012. Geo-5. Perspectivas del medio ambiente mundial. Medio ambiente para el futuro que queremos. Costa Rica. 552p.

14. POSADA A., A.; PAREDES B., A.D.; ORTIZ R., E. 2016. Enfoque sistémico aplicado al manejo de parques metropolitanos, una posición desde Bogotá D.C. - Colombia. Rev. U.D.C.A Act. \& Div. Cient. 19(1):207-217. 
15. POSADA ARRUBLA, A.; BERROCAL, A.E. 2015. Reconfiguración regional en el marco del ordenamiento territorial; Francia, un espejo para Colombia. Bogotá, Colombia. Rev. U.D.C.A Act \& Div. Cient. 18(1): 271281.

16. PROGRAMA DE LAS NACIONES UINIDAS PARA LOS ASENTAMIENTOS HUMANOS -ONU Hábitat-. 2012. Estado de las ciudades de América Latina y el Caribe Rumbo a una nueva transición urbana. Brasil. ISBN 978-92-1-132469-3.
17. SANCHIS, J. 2010. Las estrategias de desarrollo local: aproximación metodológica desde una perspectiva socio-económica e integral. Valencia (España). p.147-160.

Recibido: Agosto 18 de 2016

Aceptado: Noviembre 15 de 2016

\section{Cómo citar:}

Posada Arrubla, A.; Páez Chávez, J.V. 2016. Modelo de desarrollo local para una zona de Bogotá-Colombia, relacionando la estructura ecológica principal y el proceso de expansión urbana. Rev. U.D.C.A Act. \& Div. Cient. 19(2): 445-455. 